\title{
Observing the end of cold flow accretion using halo absorption systems
}

\author{
Stewart, K R ; Kaufmann, T ; Bullock, J S ; Barton, E J ; Maller, A H ; Diemand, J ; Wadsley, J
}

\begin{abstract}
We use cosmological smoothed particle hydrodynamic simulations to study the cool, accreted gas in two Milky Way size galaxies through cosmic time to $\mathrm{z}=0$. We find that gas from mergers and cold flow accretion results in significant amounts of cool gas in galaxy halos. This cool circum-galactic component drops precipitously once the galaxies cross the critical mass to form stable shocks, $\mathrm{M}$ vir $=\mathrm{M}$ sh 1012 $\mathrm{M}$ sun. Before reaching $\mathrm{M}$ sh, the galaxies experience cold mode accretion $(\mathrm{T}<105 \mathrm{~K})$ and show moderately high covering fractions in accreted gas: $\mathrm{fc} \quad 30 \%-50 \%$ for $\mathrm{R}<50$ comoving kpc and $\mathrm{N}_{H i}>10^{16} \mathrm{~cm}-$ 2.Thesevaluesareconsiderablylowerthanobservedcoveringfractions, suggestingthatout flowinggas(notincludedhere)is
\end{abstract}

DOI: https://doi.org/10.1088/2041-8205/735/1/L1

Posted at the Zurich Open Repository and Archive, University of Zurich

ZORA URL: https://doi.org/10.5167/uzh-54415

Journal Article

Published Version

Originally published at:

Stewart, K R; Kaufmann, T; Bullock, J S; Barton, E J; Maller, A H; Diemand, J; Wadsley, J (2011). Observing the end of cold flow accretion using halo absorption systems. Astrophysical Journal Letters, 735(1):L1.

DOI: https://doi.org/10.1088/2041-8205/735/1/L1 


\title{
OBSERVING THE END OF COLD FLOW ACCRETION USING HALO ABSORPTION SYSTEMS
}

\author{
Kyle R. Stewart ${ }^{1,8}$, Tobias Kaufmann ${ }^{2}$, James S. Bullock ${ }^{3,4}$, Elizabeth J. Barton ${ }^{3,4}$, \\ Ariyeh H. MAlleR ${ }^{5}$, Jürg Diemand ${ }^{6}$, AND James WADSley ${ }^{7}$ \\ ${ }^{1}$ Jet Propulsion Laboratory, Pasadena, CA 91109, USA \\ 2 Institute for Astronomy, ETH Zurich, CH-8093 Zurich, Switzerland \\ ${ }^{3}$ Center for Cosmology, Department of Physics and Astronomy, The University of California at Irvine, Irvine, CA 92697, USA \\ ${ }^{4}$ Center for Galaxy Evolution, Department of Physics and Astronomy, The University of California at Irvine, Irvine, CA 92697, USA \\ ${ }^{5}$ Department of Physics, New York City College of Technology, 300 Jay St., Brooklyn, NY 11201, USA \\ ${ }^{6}$ Institute for Theoretical Physics, University of Zurich, 8057 Zurich, Switzerland \\ ${ }^{7}$ Department of Physics and Astronomy, McMaster University, Main Street West, Hamilton L85 4M1, Canada \\ Received 2010 December 9; accepted 2011 April 26; published 2011 June 3
}

\begin{abstract}
We use cosmological smoothed particle hydrodynamic simulations to study the cool, accreted gas in two Milky Way size galaxies through cosmic time to $z=0$. We find that gas from mergers and cold flow accretion results in significant amounts of cool gas in galaxy halos. This cool circum-galactic component drops precipitously once the galaxies cross the critical mass to form stable shocks, $M_{\mathrm{vir}}=M_{\mathrm{sh}} \sim 10^{12} M_{\odot}$. Before reaching $M_{\mathrm{sh}}$, the galaxies experience cold mode accretion $\left(T<10^{5} \mathrm{~K}\right)$ and show moderately high covering fractions in accreted gas: $f_{c} \sim 30 \%-50 \%$ for $R<50$ comoving kpc and $N_{\mathrm{H}_{\mathrm{I}}}>10^{16} \mathrm{~cm}^{-2}$. These values are considerably lower than observed covering fractions, suggesting that outflowing gas (not included here) is important in simulating galaxies with realistic gaseous halos. Within $\sim 500 \mathrm{Myr}$ of crossing the $M_{\mathrm{sh}}$ threshold, each galaxy transitions to hot mode gas accretion, and $f_{c}$ drops to $\sim 5 \%$. The sharp transition in covering fraction is primarily a function of halo mass, not redshift. This signature should be detectable in absorption system studies that target galaxies of varying host mass, and may provide a direct observational tracer of the transition from cold flow accretion to hot mode accretion in galaxies.
\end{abstract}

Key words: cosmology: theory - galaxies: evolution - galaxies: formation - galaxies: halos - methods: numerical

Online-only material: color figures

\section{INTRODUCTION}

Recent advances in galaxy formation theory have emphasized the importance of cool gas accretion onto galaxies: gas that never shock-heats to the virial temperature of the halo (e.g., Birnboim \& Dekel 2003; Dekel \& Birnboim 2006; Kereš et al. 2009). Under this picture of galaxy formation, the cooling time of gas entering halos less massive than a critical threshold, $M_{\mathrm{vir}}=M_{\mathrm{sh}}$, is too short to sustain compressive shocks, with $M_{\text {sh }} \sim 10^{11.5}-10^{12} M_{\odot}$. Galaxies within dark matter halos less massive than $M_{\mathrm{sh}}$ experience "cold mode" accretion, since baryonic accretion onto these galaxies is dominated by cool gas. Galaxies above this transition experience "hot mode" accretion, with infalling gas shock-heating to the virial temperature. In practice, the precise value of $M_{\mathrm{sh}}$ depends somewhat on definition. In what follows we are interested in the absolute shut-down mass, when almost no cool accreted gas reaches the central regions of the halo. Thus, we adopt $M_{\mathrm{sh}} \sim 10^{12} M_{\odot}$, motivated by Dekel \& Birnboim (2006) for shocks at large fractions of the virial radius for $Z \sim 0.1$ metallicity gas. ${ }^{9}$

Unfortunately, there are currently no definitive observational tests to detect cosmological cool gas accretion. On the contrary, numerous observational studies of cool halo gas around galaxies have emphasized the presence of gas outflows, not inflows (e.g., Steidel et al. 1996, 2010; Shapley et al. 2003; Weiner et al. 2009; Rubin et al. 2011). The stark contrast between theory and

\footnotetext{
8 NASA Postdoctoral Program Fellow.

9 Kereš et al. (2009) find that less than half of a galaxy's gas is accreted from cold mode accretion for $M_{\text {vir }} \gtrsim 2 \times 10^{11} M_{\odot}$; this fraction drops to near zero at $10^{12} M_{\odot}$. Above $\sim 10^{12} M_{\odot}$ cold mode accretion is negligible.
}

observations is understandable at high redshift, as gas inflow to galaxies at $z>2$ is expected to flow along dense filaments, resulting in small global covering fractions (Faucher-Giguere \& Keres 2011; Kimm et al. 2011). In addition, galaxies at $z \sim 2$ are at the peak of cosmological star formation (Hopkins \& Beacom 2006); one might expect feedback processes to dominate any observational indicator of gas accretion at these epochs.

At lower redshifts star formation rates decline and the gaseous halos of galaxies are observed as quasar absorption systems (e.g., Bergeron \& Boissé 1991; Bowen et al. 1995; Churchill et al. 1996; Barton \& Cooke 2009; Chen et al. 2010; Gauthier et al. 2010). These studies are primarily of metal lines (Mg II, Civ, O vi, etc.) though with the Cosmic Origins Spectrograph on Hubble Space Telescope we expect Ly $\alpha$ observations to increase. In this Letter, we utilize cosmological hydrodynamic simulations to study cool gas accretion and the possibility of detecting it as quasar absorption systems. To correctly produce metal lines in a simulation requires radiative transfer, metal diffusion, and modeling of local ionizing sources; however, we are focusing on the qualitative behavior of halo gas, so we will instead give results in terms of $\mathrm{H}$ I column density calculated in the optically thin limit, without local sources. For column densities below the Lyman limit $\left(2 \times 10^{17} \mathrm{~cm}^{-2}\right)$ this should be fairly robust, but for higher column densities we expect a full treatment would lead to quantitative but not qualitative differences.

\section{SIMULATIONS AND ANALYSIS}

\subsection{The Simulations}

Our two simulations utilize separate sets of cosmological initial conditions, with each simulation tracking the evolution 
of a roughly Milky Way size dark matter halo until $z=0$. We refer to these two simulations as "Halo 1" and "Halo 2," since we primarily investigate the properties of the single most massive galaxy in each simulation. Halo 1 has an active merger history until $z \sim 1.5$, but subsequently experiences a relatively quiescent merger history (a dark matter only simulation of the same initial conditions was performed at very high resolution in the Via Lactea II simulation of Diemand et al. 2008). In contrast, Halo 2 experiences a more quiescent early history, but has a major merger at $z \sim 0.8$. For both simulations, the most massive galaxy has a dark halo mass of $M_{\text {vir }}(z=0)=1.4 \times 10^{12} M_{\odot}$, and is positioned within a large scale filament of dark matter and gas, typical for galaxies of this mass.

We use multiple mass particle grid initial conditions generated with the GRAFIC-2 package (Bertschinger 2001) and the bestfit cosmological parameters of the WMAP three-year data release (Spergel et al. 2007): $\Omega_{M}=0.238, \Omega_{\Lambda}=0.762$, $H_{0}=73 \mathrm{~km} \mathrm{~s}^{-1} \mathrm{Mpc}^{-1}, n_{s}=0.951$, and $\sigma_{8}=0.74$. We implement the "zoom in" technique to properly account for large-scale tidal torques as described in Katz \& White (1993), and each simulation is contained within a periodic box of 40 comoving $\mathrm{Mpc}$, with the highest resolution region limited to a $\sim 6$ comoving Mpc cube. We use the smoothed particle hydrodynamics (SPH) code GASOLINE (Wadsley et al. 2004). In the highest resolution region of each simulation, the masses of the simulated particles in the initial conditions are: $\left(m_{\text {dark }}, m_{\text {gas }}\right)=(17,3.7) \times 10^{5} M_{\odot}$, with a force softening of $332 \mathrm{pc}$.

The code assumes a uniform UV background from QSO, implemented following Haardt \& Madau (1996) and F. Haardt (2002, private communication). It implements star formation, as well as Compton and radiative cooling, as described in Katz et al. (1996), calculating the abundance of neutral hydrogen by assuming an optically thin ideal gas of primordial composition and in ionization equilibrium with the UV background, treating collisional ionization, photoionization, and recombination processes. We do not include full treatment of radiative transfer. Because we primarily focus on qualitative attributes of cool halo gas and its evolution with time, we do not believe this will significantly impact our results. We expect that a full treatment would increase the amount of very high density gas, causing a progressively larger increase to our reported covering fractions, starting at a few times the Lyman limit.

Type II supernovae are modeled using an analytical treatment of blastwaves, creating turbulent motions in nearby gas particles that keeps them from cooling and forming stars, as described in Stinson et. al (2006). This feedback model results in minimal winds of $\sim 100 \mathrm{~km} \mathrm{~s}^{-1}$ that mostly affect hot gas and are more prominent in $M_{\text {vir }} \lesssim 10^{11} M_{\odot}$ halos (Shen et al. 2010). For the mass range considered in this Letter, this feedback model does not result in cool outflows that would be detectable through absorption. The only two free parameters in the star formation and feedback model have been fixed in order to produce galaxies with a realistic star formation rates, disk thicknesses, gas turbulence, and Schmidt law over a range in dynamic masses (Governato et al. 2007). We note our feedback model is very similar to those used in recent simulations that have shown great success in producing realistic disk-type galaxies (Governato et al. 2009), as well as matching the mass-metallicity relation (Brooks et al. 2007) and the abundance of damped Ly $\alpha$ systems at $z=3$ (Pontzen et al. 2008). We refer the reader to Governato et al. (2009) for a more detailed description of the simulation code. At each output snapshot of our simulation, we define the virial radius by Bryan \& Norman (1998), noting that this is a fairly typical definition used in $\mathrm{N}$-body simulations of dark matter substructure (e.g., Stewart et al. 2008).

While a detailed morphological analysis of our simulated galaxies is beyond the scope of this Letter, visual inspection shows that both galaxies are disks before they reach the transition mass for hot mode accretion. After this transition, Halo 1 remains roughly disk-like but grows a massive bulge as hot gas cools from the halo, similar to the most massive galaxy in Governato et al. (2007) and Brooks et al. (2009). Due to the major merger in Halo 2 just prior to reaching the transition mass, it develops into a more spheroidal galaxy. We note that previous works that have produced disk galaxies at $z=0$, even for galaxies with major mergers at $z<1$, typically involve halos less massive than those we study here (e.g., $7 \times 10^{11} M_{\odot}$ in Governato et al. 2009), which may be the cause of these morphological differences.

\subsection{Analysis}

To study the cool halo gas in our simulated galaxies, we analyze 794 regularly spaced sight lines within 100 comoving $\mathrm{kpc}$, for three orthogonal orientations of each galaxy. For each sight line, we compute the column density of neutral hydrogen per unit velocity, as a function of velocity along the line of sight, mimicking the practice of searching for absorption gas along a quasar line of sight. We do this by dividing each sight line into spatial bins of order the size of the spatial resolution of the simulation. For each spatial bin, each gas particle with a smoothing length that intersects the line of sight contributes to the total mass density of the bin. For each of these particles, the contribution of the spline kernel to the column density of each bin that overlaps the kernel is integrated along the line of sight. The velocity of each bin is the mass-weighted velocity of particles contributing to the total column density of neutral hydrogen.

We define the covering fraction of accreted neutral hydrogen, $f_{c}(<R)$, as the total fraction of sight lines (within a radius $R$ from the center of the galaxy) for which $N_{\mathrm{HI}}>10^{16.0}-10^{18.7} \mathrm{~cm}^{-2}$. For $>95 \%$ of cases, the full width at half-maximum spread in velocities of absorbing sightlines ${ }^{10}$ is $<110 \mathrm{~km} \mathrm{~s}^{-1}$. We choose these column density limits in order to probe different types of cool gaseous structures that may exist in the halos of galaxies, noting that they correspond to a plausible minimum threshold for halo gas detection via Mg II absorption (Rigby et al. 2002; Churchill et al. 2000).

Some past studies of metal line absorption systems associated with galaxies suggest that there may be a critical radius, outside of which cool gas clouds cannot form (e.g., Chen et al. 2001, 2010), while a study by Steidel et al. (2010) suggests that $f_{c}(<R)$ decays as a power law in $R$. Based on the typical extent of neutral hydrogen in our galaxies, we present covering fractions in terms of three fixed radii: $R<30,50$, and $100 \mathrm{co}-$ moving kpc (for a detailed exploration of the radial dependence of $f_{c}(<R)$, see Stewart et al. 2011). We include the galaxy disk in our analysis, but its contribution to the covering fraction is relatively minor. For $R<30,50$, and 100 comoving kpc, $f_{c}$ for the disk is $<10 \%, 4 \%$, and $1 \%$, respectively.

\footnotetext{
10 Because our feedback model does not produce cool gas outflows, our absorption sight lines are never offset from the systemic velocity by more than $1000 \mathrm{~km} \mathrm{~s}^{-1}$.
} 

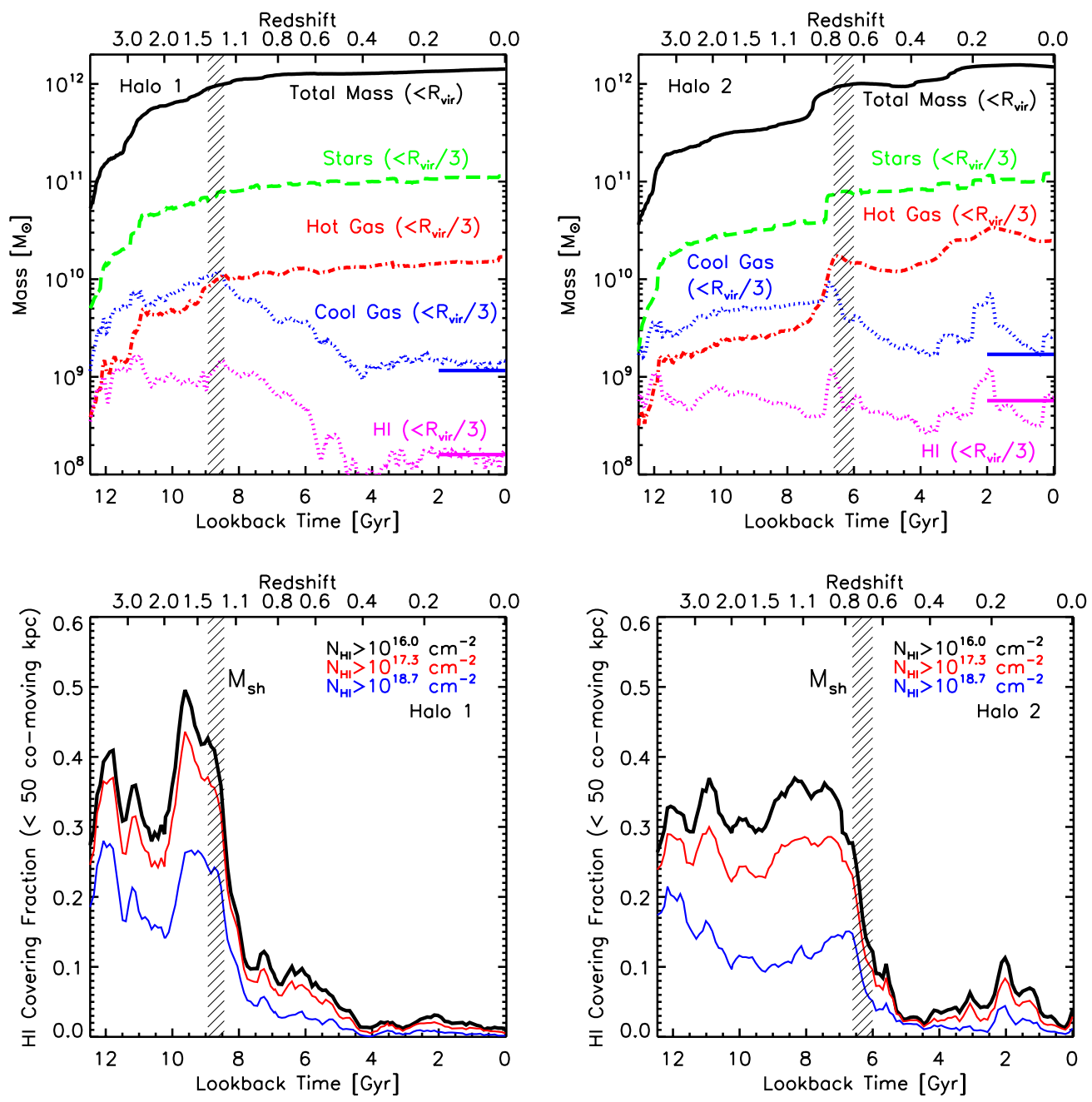

Figure 1. Masses and covering fractions of each galaxy vs. look-back time. The left panels show Halo 1, while the right panels show Halo 2 . Top: the total virial mass (solid black), and baryonic masses within $R<R_{\mathrm{vir}} / 3$ ( $\simeq 100 \mathrm{kpc}$ at $z=0$ for halos of this mass). Stellar mass, hot gas mass $\left(T>10^{5} \mathrm{~K}\right)$, cool gas mass $\left(T \leqslant 10^{5} \mathrm{~K}\right)$, and neutral hydrogen are given by the dashed green, dash-dot red, dotted blue, and dotted magenta lines, respectively. The thick horizonal lines on the right side of each plot show the cool gas mass and neutral hydrogen contained within the central galaxy and massive satellite galaxies at $z=0$. Bottom: covering fraction of neutral hydrogen within $R<50$ comoving kpc as a function of time. Black, red, and blue lines represent minimum column densities $N_{\mathrm{H}_{\mathrm{I}}}>10^{16.0}, 10^{17.3}$, and $10^{18.7} \mathrm{~cm}^{-2}$, respectively. The vertical hashed bar in each panel shows when the galaxy transitions from cold mode to hot mode accretion (see Section 4).

(A color version of this figure is available in the online journal.)

\section{GALAXY GROWTH AND EVOLUTION}

Figure 1 shows the mass accretion history of our galaxies, as well as the covering fraction of each galaxy over time (Halo 1 on the left, Halo 2 on the right). In the top panels, the virial mass is given by the solid black line, while other curves give baryonic masses within $R_{\mathrm{vir}} / 3(100 \mathrm{kpc}$ at $z=0)$. The stellar mass, hot gas mass $\left(T>10^{5} \mathrm{~K}\right)$, cool gas mass $\left(T \leqslant 10^{5} \mathrm{~K}\right)$, and neutral hydrogen mass are given by the dashed green, dotdashed red, dotted blue, and dotted magenta lines, respectively. Halo 1 experiences several large mergers at $z>1.5$, with a relatively quiescent growth after $z=1$, while Halo 2 has a more quiescent early growth $(z<1)$, but experiences a major merger at $z \sim 0.8$, and another moderately large merger at $z \sim 0.2$. These signatures, especially the late-time mergers for Halo 2, can be seen by the sharp increases in various curves in the top panels. Note that most of the halo gas within $R<R_{\text {vir }} / 3$ is cool until the halo grows to $M_{\text {vir }} \sim 10^{12} M_{\odot}$. (See Section 4 for more on this transition. The vertical hashed bar in each panel shows the range where $M_{\mathrm{vir}}=(0.9-1.0) \times 10^{12} M_{\odot}$ for each galaxy. $)$ For proper context, we also include the cool gas mass enclosed within the main galaxy $(R<10 \mathrm{kpc})$ or in massive satellite galaxies at $z=0$, represented by the thick horizontal lines at the right side of each plot. It is clear, then, that the vast majority of cool gas is associated with galaxies at late times and is not spread throughout the halo. In contrast, when the galaxy first reaches the transition mass, the cool gas in the galactic disk is only $\sim 10^{9} M_{\odot}$, and does not dominate the cool gas mass within $R_{\mathrm{vir}} / 3$.

The bottom panels of Figure 1 show the time evolution of $f_{c}(R<50$ comoving $\mathrm{kpc})$ for each galaxy, with different curves representing different column densities ${ }^{11}: N_{\mathrm{H}_{\mathrm{I}}}>10^{16.0} \mathrm{~cm}^{-2}$ (thick black), $N_{\mathrm{HI}_{\mathrm{I}}}>10^{17.3} \mathrm{~cm}^{-2}$ (thin red), and $N_{\mathrm{HI}_{\mathrm{I}}}>$ $10^{18.7} \mathrm{~cm}^{-2}$ (thin blue). In general, both galaxies show remarkably flat evolution of covering fraction with time (for $M_{\mathrm{vir}}<M_{\mathrm{sh}}$ ), with $f_{c} \sim 30 \%-40 \%$ for $z<4$. In detail, each galaxy's covering fraction varies with recent merger history, as demonstrated by the sudden spikes and dips in Figure 1. These

\footnotetext{
${ }^{11}$ Note that the higher column density lines will not be correct, in detail, because we do not have full treatment of radiative transfer in our analysis. Our estimates are likely too low for higher density gas.
} 

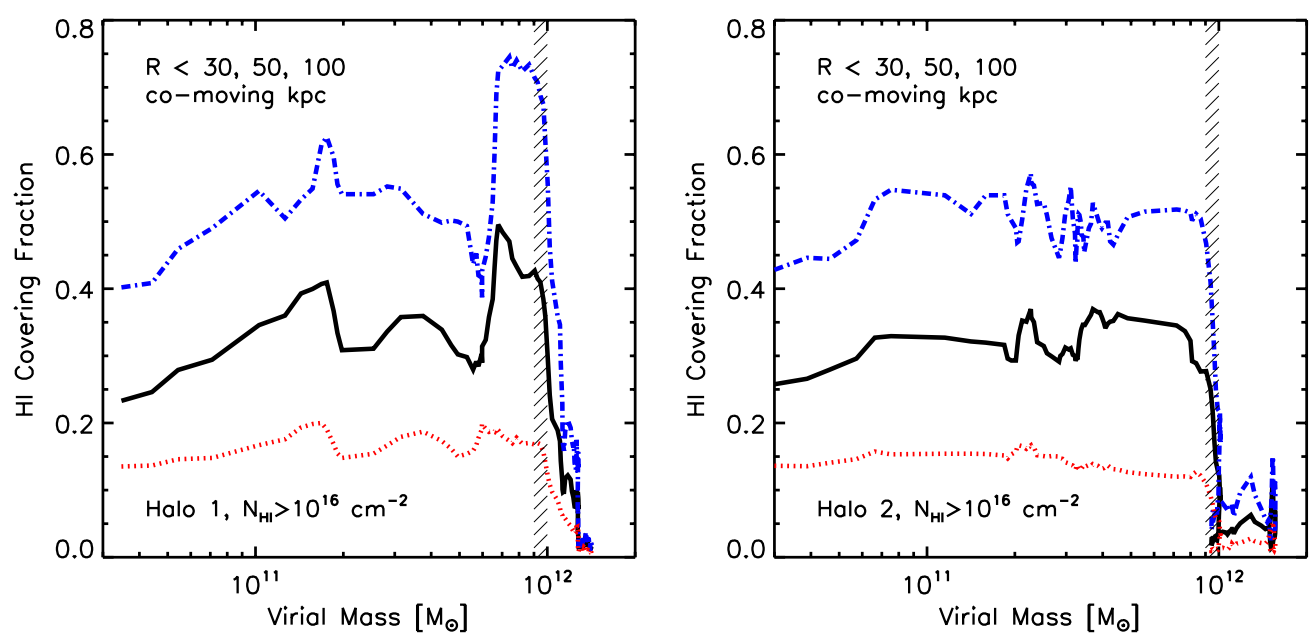

Figure 2. Covering fraction of neutral hydrogen as a function of the halo virial mass. The left and right panels show Halo 1 and Halo 2. Different lines correspond to outer radii of $R<30,50$, and 100 comoving kpc (dot-dashed blue, solid black, dotted red, respectively). Note the drastic transition in covering fraction at the cold/hot mode transition of $M_{\mathrm{vir}}=M_{\mathrm{sh}} \sim 10^{12} M_{\odot}$, which occurs at $z \sim 1.3$ for Halo 1 and $z \sim 0.8$ for Halo 2 .

(A color version of this figure is available in the online journal.)

variations are primarily caused by mergers, which play an important role in cumulative gas accretion onto galaxies as well as halos (especially at $z>1$, when mergers are more frequent and more gas-rich than at late times; see, e.g., Stewart et al. 2009a, 2009b). We note that Kacprzak et al. (2010) have carried out a similar investigation to what we present here, and also found an accretion-related origin for many $\mathrm{Mg}$ II absorption systems. In addition, Kacprzak et al. (2007) found a correlation between galaxy asymmetry and the presence of halo gas, again suggesting a link between gas accretion and halo absorption systems. Reassuringly, our covering fractions at high redshift are in qualitative agreement with a high-redshift simulation by Faucher-Giguere \& Keres (2011), utilizing a different SPH code and including treatment of radiative transfer. For similar mass galaxies as our own, they quote an average covering fraction of $\sim 20 \%-30 \%$ at $z=2-4\left(R<R_{\text {vir }} / 2\right.$ and $N_{\text {H }}>10^{17.2} \mathrm{~cm}^{-2}$ ).

We emphasize that these moderate covering fractions are a result of gas accretion only. Our feedback model does not produce significant outflows. We do this both to achieve a robust result (the physical mechanism behind galaxy outflows is largely uncertain) and to focus on signatures of cold flow accretion. Since galactic outflows should populate the halo with additional cool gas, our results serve as a lower limit on the true covering fractions of galaxies.

\section{COLD FLOWS AND COVERING FRACTIONS}

Observed covering fractions typically vary from $\sim 50 \%$ (e.g., Tripp \& Bowen 2005; Chen \& Mulchaey 2009) to near unity (e.g., Chen et al. 2001; Chen \& Tinker 2008), but there are indications that more massive galaxies (LRGs) may have systematically lower values $(\sim 10 \%-15 \%$; Gauthier et al. 2010; Bowen $\&$ Chelouche 2011). Despite this variation in the literature, our findings are significantly lower than those observed, suggesting that outflows are a required component in producing galaxies with realistic gaseous halos. Still, the contribution from accreted gas presented here is significant, so that the sudden drop in $f_{c}$ (Figure 1) should be observable in real galaxies.

Keeping in mind the theoretical framework of cold and hot mode accretion (see Section 1), Figure 2 shows the covering fraction as a function of halo virial mass rather than time, for three choices of outer radii: 30, 50, and 100 comoving kpc (dotdashed blue, solid black, and dotted red lines, respectively). Regardless of the radius used, $f_{c}(<R)$ declines sharply at $M_{\text {vir }}=M_{\text {sh }}$, when cold mode accretion ends for each galaxy. ${ }^{12}$

A preliminary analysis suggests that the timescale for cool halo gas to fall onto the central galaxy is short (K. R. Stewart et al. 2012, in preparation); thus the reason for this sharp decline in covering fraction becomes clear. Once the galaxy crosses the threshold into hot mode accretion, the existing cool halo gas quickly sinks onto the galaxy and cannot be replaced by subsequent gas accretion. A galaxy in this hot mode accretion can only maintain a cool gaseous halo by mechanisms other than direct accretion. For example, outflowing winds may be capable of re-distributing cool gas from the galaxy into the halo. Alternatively, galaxy halos can form instability-triggered clouds, resulting in cool gas that is neither freshly accreted nor outflowing (e.g., Maller \& Bullock 2004; Kaufmann et al. 2009; Kereš \& Hernquist 2009). ${ }^{13}$

This picture of gaseous halo formation has direct consequences on observations of halo absorption systems. We predict that galaxies above the transition mass have drastically smaller covering fractions within a fixed comoving radius, with respect to accreted cool gas. While we have presented a small range in values for $M_{\mathrm{sh}}$, different models vary in detail. Typically, $M_{\text {sh }} \sim 10^{11.5}-10^{12.5} M_{\odot}$, corresponding to central galaxies with stellar masses $M_{\text {star }} \sim 10^{10.2}-10^{10.8} M_{\odot}$ at $z=0$, or $M_{\text {star }} \sim 10^{9.8}-10^{10.8} M_{\odot}$ at $z=1$ (based on abundance matching, e.g., Conroy \& Wechsler 2009). If moderately high covering fractions are found to exist for more massive galaxies (which are not undergoing gas-rich mergers), that gas is likely the result of outflows, and should show distinct differences in metallicity and kinematics when compared to freshly accreted cool gas (for an observable kinematic signature of cool accreted gas; see Stewart et al. 2011). We note that Tinker \& Chen (2008) found observational evidence from correlation functions that supports this

\footnotetext{
12 To emphasize that a precise value of $M_{\mathrm{sh}}$ is uncertain, the vertical hashed bar in each panel shows a small range of values, $M_{\mathrm{sh}}=(0.9-1) \times 10^{12} M_{\odot}$. 13 While our resolution does not allow us to directly simulate these clouds, we do not expect high covering fractions due to cloud fragmentation, for standard hot gas profiles (Kaufmann et al. 2009).
} 
model of a cold/hot mode transition influencing the presence of halo absorption systems.

\section{CONCLUSION}

We have used two high-resolution, cosmological SPH simulations as a tool for studying the cool gaseous halos of galaxies. By creating mock observation sight lines, representing cool gas detection via absorption, we present the covering fraction of neutral hydrogen in our galaxies over time. Our primary results are summarized as follows.

1. To first order, the covering fraction of accreted cool gas is relatively stable at $f_{c} \sim 30 \%-40 \%$ ( $R<50$ comoving $\mathrm{kpc}, N_{\mathrm{HI}}>10^{16} \mathrm{~cm}^{-2}$ ), as long as the galaxy continues to accrete cool gas from the cosmic web.

2. As soon as our simulated galaxies cross the threshold between cold mode accretion and hot mode accretion (when the halo is massive enough to support stable shocks at a large fraction of the virial radius, $M_{\text {vir }} \sim 10^{12} M_{\odot}$ ), the lack of cool accreted gas results in a suppression of cool halo gas. Within $~ 500 \mathrm{Myr}$ of reaching this threshold mass, the covering fraction drops from $30 \%-50 \%$ to $5 \%-10 \%$. A transition this sharp should be directly observable via metal line absorption system studies.

We have used a feedback model without cool gas outflows here, focusing on galaxy halo properties that are a natural consequence of cosmological gas accretion in LCDM. However, observations have shown that galaxy outflows are an abundant phenomenon, and likely play an important role in shaping the properties of cool gaseous halos around galaxies. We believe future work in comparing observations to a variety of simulations with different feedback models would prove a valuable tool in testing theoretical models of galaxy formation, as well as understanding the underlying nature of galaxy halo observations.

The simulations presented here were run on the Cosmos cluster at JPL, and the Greenplanet cluster at UCI. K.R.S. thanks all those who commented on a preliminary draft of this Letter. This research was carried out at the Jet Propulsion Laboratory, California Institute of Technology, under a contract with the National Aeronautics and Space Administration. K.R.S. is supported by an appointment to the NASA Postdoctoral Program at the Jet Propulsion Laboratory, administered by Oak Ridge Associated Universities through a contract with NASA. J.S.B. and K.R.S. were partially supported by NASA grant NNX09AG01G. T.K. and J.D. were supported by the Swiss National Science Foundation (SNF).

\section{REFERENCES}

Barton, E. J., \& Cooke, J. 2009, AJ, 138, 1817 Bergeron, J., \& Boissé, P. 1991, A\&A, 243, 344 Bertschinger, E. 2001, ApJS, 137, 1
Birnboim, Y., \& Dekel, A. 2003, MNRAS, 345, 349

Bowen, D. V., Blades, J. C., \& Pettini, M. 1995, ApJ, 448, 634

Bowen, D. V., \& Chelouche, D. 2011, ApJ, 727, 47

Brooks, A. M., Governato, F., Booth, C. M., Willman, B., Gardner, J. P., Wadsley, J., Stinson, G., \& Quinn, T. 2007, ApJ, 655, L17

Brooks, A. M., Governato, F., Quinn, T., Brook, C. B., \& Wadsley, J. 2009, ApJ, 694, 396

Bryan, G. L., \& Norman, M. L. 1998, ApJ, 495, 80

Chen, H., Lanzetta, K. M., \& Webb, J. K. 2001, ApJ, 556, 158

Chen, H., \& Mulchaey, J. S. 2009, ApJ, 701, 1219

Chen, H., \& Tinker, J. L. 2008, ApJ, 687, 745

Chen, H., Wild, V., Tinker, J. L., Gauthier, J., Helsby, J. E., Shectman, S. A., \& Thompson, I. B. 2010, ApJ, 724, L176

Churchill, C. W., Mellon, R. R., Charlton, J. C., Jannuzi, B. T., Kirhakos, S., Steidel, C. C., \& Schneider, D. P. 2000, ApJS, 130, 91

Churchill, C. W., Steidel, C. C., \& Vogt, S. S. 1996, ApJ, 471, 164

Conroy, C., \& Wechsler, R. H. 2009, ApJ, 696, 620

Dekel, A., \& Birnboim, Y. 2006, MNRAS, 368, 2

Diemand, J., Kuhlen, M., Madau, P., Zemp, M., Moore, B., Potter, D., \& Stadel J. 2008, Nature, 454, 735

Faucher-Giguere, C.-A., \& Keres, D. 2011, MNRAS, 412, L118

Gauthier, J., Chen, H., \& Tinker, J. L. 2010, ApJ, 716, 1263

Governato, F., Willman, B., Mayer, L., Brooks, A., Stinson, G., Valenzuela, O., Wadsley, J., \& Quinn, T. 2007, MNRAS, 374, 1479

Governato, F., et al. 2009, MNRAS, 398, 312

Haardt, F., \& Madau, P. 1996, ApJ, 461, 20

Hopkins, A. M., \& Beacom, J. F. 2006, ApJ, 651, 142

Kacprzak, G. G., Churchill, C. W., Ceverino, D., Steidel, C. C., Klypin, A., \& Murphy, M. T. 2010, ApJ, 711, 533

Kacprzak, G. G., Churchill, C. W., Steidel, C. C., Murphy, M. T., \& Evans, J. L. 2007, ApJ, 662, 909

Katz, N., Weinberg, D. H., \& Hernquist, L. 1996, ApJS, 105, 19

Katz, N., \& White, S. D. M. 1993, ApJ, 412, 455

Kaufmann, T., Bullock, J. S., Maller, A. H., Fang, T., \& Wadsley, J. 2009, MNRAS, 396, 191

Kereš, D., \& Hernquist, L. 2009, ApJ, 700, L1

Kereš, D., Katz, N., Fardal, M., Davé, R., \& Weinberg, D. H. 2009, MNRAS, 395,160

Kimm, T., Slyz, A., Devriendt, J., \& Pichon, C. 2011, MNRAS, 413, L51

Maller, A. H., \& Bullock, J. S. 2004, MNRAS, 355, 694

Pontzen, A., et al. 2008, MNRAS, 390, 1349

Rigby, J. R., Charlton, J. C., \& Churchill, C. W. 2002, ApJ, 565, 743

Rubin, K. H. R., Prochaska, J. X., Ménard, B., Murray, N., Kasen, D., Koo, D. C., \& Phillips, A. C. 2011, ApJ, 728, 55

Shapley, A. E., Steidel, C. C., Pettini, M., \& Adelberger, K. L. 2003, ApJ, 588, 65

Shen, S., Wadsley, J., \& Stinson, G. 2010, MNRAS, 407, 1581

Spergel, D. N., et al. 2007, ApJS, 170, 377

Steidel, C. C., Erb, D. K., Shapley, A. E., Pettini, M., Reddy, N., Bogosavljević, M., Rudie, G. C., \& Rakic, O. 2010, ApJ, 717, 289

Steidel, C. C., Giavalisco, M., Pettini, M., Dickinson, M., \& Adelberger, K. L. 1996, ApJ, 462, L17

Stewart, K. R., Bullock, J. S., Barton, E. J., \& Wechsler, R. H. 2009a, ApJ, 702, 1005

Stewart, K. R., Bullock, J. S., Wechsler, R. H., \& Maller, A. H. 2009b, ApJ, 702, 307

Stewart, K. R., Bullock, J. S., Wechsler, R. H., Maller, A. H., \& Zentner, A. R. 2008, ApJ, 683, 597

Stewart, K. R., Kaufmann, T., Bullock, J. S., Barton, E. J., Maller, A. H., Diemand, J., \& Wadsley, J. 2011, ApJ, submitted (arXiv:1103.4388)

Stinson, et al. 2006, MNRAS, 373, 1074

Tinker, J. L., \& Chen, H. 2008, ApJ, 679, 1218

Tripp, T. M., \& Bowen, D. V. 2005, in IAU Colloq. 199: Probing Galaxies through Quasar Absorption Lines, ed. P. Williams, C.-G. Shu, \& B. Menard (Cambridge: Cambridge Univ. Press), 5

Wadsley, J. W., et al. 2004, New Astron., 9, 137

Weiner, B. J., et al. 2009, ApJ, 692, 187 